\title{
A Parameter Property of Classical Solutions of Cauchy Problems
}

\author{
Min He \\ Kent State University at Trumbull, Warren, $\mathrm{OH} 44483$, USA \\ Correspondence should be addressed to Min He; mhe@kent.edu
}

Received 1 November 2013; Accepted 26 December 2013; Published 10 February 2014

Academic Editor: Yuming Xing

Copyright (C) 2014 Min He. This is an open access article distributed under the Creative Commons Attribution License, which permits unrestricted use, distribution, and reproduction in any medium, provided the original work is properly cited.

This work is concerned with the abstract Cauchy problems that depend on parameters. The goal is to study continuity in the parameters of the classical solutions of the Cauchy problems. The situation considered in this work is when the operator of the Cauchy problem is not densely defined. By applying integrated semigroup theory and the results on continuity in the parameters of $C_{0}$-semigroup and integrated semigroup, we obtain the results on the existence and continuity in parameters of the classical solutions of the Cauchy problems. The application of the obtained abstract results in a parabolic partial differential equation is discussed in the last section of the paper.

\section{Introduction}

Many dynamical systems [1-4] such as differential equations, integrodifferential equations, and functional differential equations involve parameters in their equations and/or their boundary conditions. For instance, consider the parabolic partial differential equation

$$
\begin{gathered}
u_{t}=u_{x x}+k_{1} u_{x}+k_{2} u, \quad \text { for } t \geq 0, k_{1}, k_{2} \in R, \\
u(x, 0)=u_{0}(x), \quad \text { for } x \in[0,1], \\
u(0, t)=u(1, t)=0,
\end{gathered}
$$

where $k_{1}$ and $k_{2}$ are considered as parameters.

When making the change in variable $v=e^{\left(k_{1} / 2\right) x} u$, we have that $v$ satisfies the equation

$$
\begin{gathered}
v_{t}=v_{x x}+\left(k_{2}-\frac{k_{1}^{2}}{4}\right) v, \quad \text { for } t \geq 0, k_{1}, k_{2} \in R, \\
v(x, 0)=v_{0}(x), \quad \text { for } x \in[0,1], \\
v(0, t)=v(1, t)=0,
\end{gathered}
$$

where $v_{0}(x)=e^{\left(k_{1} / 2\right) x} u_{0}(x)$.

Then, the abstract Cauchy problem formulated from (2) is

$$
\frac{d v(t)}{d t}=A(\varepsilon) v(t), \quad v(0)=v_{0}
$$

on $X=\left(C[0,1],\|\cdot\|_{\infty}\right), t \geq 0$, where

$$
\begin{gathered}
A(\varepsilon)=\frac{d^{2}}{d x^{2}}+B(\varepsilon), \quad \text { where } B(\varepsilon)=\left(k_{2}-\frac{k_{1}^{2}}{4}\right), \\
\varepsilon=\left(k_{1}, k_{2}\right) \in R^{2}, \\
D(A(\varepsilon))=\left\{v \in C^{2}[0,1] \mid v(0)=v(1)=0\right\} .
\end{gathered}
$$

Equation (3) shows that the operator is dependent on the parameter $\varepsilon$. Thus, it is natural to investigate the effects of the parameter on the classical solutions of Cauchy problems. One of the natural questions is to determine the conditions for continuity with respect to the parameter of the classical solution of the abstract Cauchy problem

$$
\begin{gathered}
u^{\prime}(t)=A(\varepsilon) u(t)+f(t), \quad \text { for } t \geq 0, \\
u(0)=u_{0},
\end{gathered}
$$

where $\varepsilon$ is the parameter.

According to the semigroup theories, $C_{0}$-semigroup (i.e, strongly continuous semigroup) and integrated semigroup play key roles in determining parameter properties of classical or integral solutions of Cauchy problems that depend on parameters. Thus, a lot of work has focused on studying continuity in parameters of $C_{0}$-semigroup and/or integrated semigroup. Lizama [5], Nicaise [6], and Busenberg and $\mathrm{Wu}$ 
[7] have established approximation theorems for integrated semigroups concerning a one-dimensional parameter. These results may be interpreted to imply continuity in parameters of integrated semigroups. Grimmer and/or He ([2-4], and the reference therein) have made a systematic study on continuity in multiparameters of $C_{0}$-semigroup and integrated semigroup. In this paper, we will focus on determining conditions that can directly obtain the continuity in parameter of the classical solution of Cauchy problem (5). It turns out that the conditions in our theorems are directly imposed on the operator of Cauchy problem (5), which are very convenient and easy to verify. As it will be illustrated in the application of the obtained results in (1), the condition for continuity in parameter is naturally possessed by (1). And a number of equations studied in ([1-4], and the reference therein) also naturally satisfy the condition for continuity with respect to parameter.

Furthermore, (3) indicates that the operator $A(\varepsilon)$ is not densely defined. However, the theory of integrated semigroup is a powerful tool in dealing with nondensely defined operators. In Section 2, we will provide background information on integrated semigroup and state some existing theorems that will be needed for proving our main theorems. In Section 3, we apply the continuity results with respect to parameters of $C_{0}$-semigroup and integrated semigroup we derive the analogous result for the classical solution of Cauchy problem. We will begin with discussing the homogeneous Cauchy problem

$$
\begin{gathered}
u^{\prime}(t)=A(\varepsilon) u(t), \quad \text { for } t \geq 0, \\
u(0)=u_{0} .
\end{gathered}
$$

Applying the continuity results of $C_{0}$-semigroup and integrated semigroup theory, we prove that the unique classical solution of (6) is continuous with respect to parameter. Using the obtained result for (6), we will study the nonhomogeneous Cauchy problem (5) and present a theorem for continuity with respect to parameters of the classical solution of the nonhomogeneous Cauchy problem (5). In the last section, we will discuss the application of obtained abstract results in (1). As one will see, the obtained abstract result is very easy to apply.

\section{Preliminaries}

We begin with providing the background information about the integrated semigroup and state some results that will be used in the following sections.

Let $\left(X,\|\cdot\|_{X}\right)$ be a Banach space and let $P$ be an open subset of a finite-dimensional normed linear space $\mathscr{P}$ with norm $|\cdot|$.

Definition 1 (see $[8]) .\{S(t)\}_{t \geq 0} \subset \mathscr{B}(X)$ is called an integrated semigroup if
(a) $S(0)=0$,
(b) $S(t) S(\tau) x=\int_{0}^{t}(S(\tau+r)-S(r)) x d r$, for every $x \in X$,
(c) for any $x \in X, S(t) x:[0, \infty)$ is continuous.

Definition 2 (see [8]). An integrated semigroup $S$ is called nondegenerate if $S(t) x=0$ for all $t \geq 0$ implies that $x=0$.

Definition 3 (see [8]). The generator $A$ of a nondegenerate integrated semigroup $S$ is defined by letting $x, y \in X$, then $x \in D(A)$ and $A x=y$ if

$$
S(\cdot) x \in C^{1}([0, \infty), X), \quad S^{\prime}(t) x-x=S(t) y, \quad t \geq 0 .
$$

Definition 4 (see [8]). An integrated semigroup $S$ is said to be of type $(M, \omega)$, where $M \geq 1$, iff for $t, \tau \geq 0$,

$$
\|S(t+r)-S(t)\| \leq M \int_{t}^{t+r} e^{\omega s} d s .
$$

Theorem 5 (see [8]). Define $X_{0}=\overline{D(A)}$. Define the part $A_{0}$ of $A$ as

$$
A_{0}=A \quad \text { on } D\left(A_{0}\right)=\left\{u \in D(A): A u \in X_{0}\right\} \text {. }
$$

Assume that $(\lambda I-A)^{-1} \in \mathscr{B}(X)$ for all $\lambda>0$ is large and that

$$
\limsup _{\lambda \rightarrow \infty} \lambda\left\|(\lambda I-A)^{-1}\right\|<+\infty .
$$

Then $D\left(A_{0}\right)$ is dense in $X_{0}$, and if $A_{0}$ generates a $C_{0}$-semigroup on $X_{0}$, then $A$ generates a nondegenerate integrated semigroup of type $(M, \omega)$ on $X$.

In the sequel we use " $A$ is a Hille-Yosida operator" to mean that there exist $M \geq 1$ and $\omega \in R$ such that $\lambda>\omega$ implies $\lambda \in \rho(A)$ (the resolvent set of $A$ ) and

$$
\left\|(\lambda I-A)^{-n}\right\| \leq \frac{M}{(\lambda-\omega)^{n}}, \quad \text { for } \lambda>\omega, n=1,2, \ldots .
$$

Note that the boundedness of the resolvent $(\lambda I-A)^{-1}$ implies that $A$ is closed.

Theorem 6 (see [9]). The following two statements are equivalent:

(a) $A$ is the generator of a nondegenerate semigroup $S$ of type $(M, \omega)$,

(b) $A$ is a Hille-Yosida operator.

Theorem 7 (see [8]). If $A$ is a Hille-Yosida operator, then the part $A_{0}$ of $A$ in $X_{0}$ generates a $C_{0}$-semigroup $T(t)$ on $X_{0}$ satisfying

$$
\|T(t)\| \leq M e^{\omega t}
$$

Furthermore, A generates a nondegenerate integrated semigroup $S(s)$ on $X$ with

$$
\|S(t+r)-S(t)\| \leq M \int_{t}^{t+r} e^{\omega s} d s
$$


$S(t)$ is related to $T(t)$ by

$$
S(t) x=\lim _{\lambda \rightarrow \infty} \int_{0}^{t} T(s) \lambda(\lambda-A)^{-1} x d s
$$

for $t \geq 0, x \in X$. Moreover, $X_{0}=C^{1}$ and $T(t)=S^{\prime}(t)$.

Consider the nonhomogeneous Cauchy problem

$$
\begin{gathered}
u^{\prime}(t)=A u(t)+f(t), \quad \text { for } t \geq 0, \\
u(0)=u_{0},
\end{gathered}
$$

where $f:[0, \infty) \rightarrow X$ is a continuous function.

Theorem 8 (see [10]). Let $A$ be the generator of a nondegenerate integrated semigroup of type $(M, \omega) ; f$ is continuously differentiable, $x \in D(A), A x+f(0) \in X_{0}=\overline{D(A)}$. Then

$$
u(t)=S^{\prime}(t) x+\frac{d}{d t} \int_{0}^{t} S(t-\tau) f(\tau) d \tau
$$

is the unique classical solution to (15); that is $u$ is the only continuously differentiable function with values in $D(A)$ such that (15) is satisfied.

\section{Continuity in Parameter of Classical Solutions of Cauchy Problems}

We first study the homogeneous abstract Cauchy problem

$$
\begin{gathered}
u^{\prime}(t)=A(\varepsilon) u(t), \quad \text { for } t \geq 0, \\
u(0)=u_{0},
\end{gathered}
$$

where $\varepsilon$ is the parameter in $P$. For each $\varepsilon \in P$, the operator $A(\varepsilon)$ is a closed linear and a nondensely defined operator on the Banach space $X$.

For each $\varepsilon \in P$, define the part $A_{0}(\varepsilon)$ of $A(\varepsilon)$ as

$$
\begin{aligned}
& \begin{array}{l}
A_{0}(\varepsilon) \\
\text { on } D\left(A_{0}(\varepsilon)\right) \\
\qquad=\left\{u \in D(A(\varepsilon)): A(\varepsilon) u \in X_{0}(\varepsilon)=\overline{D(A(\varepsilon))}\right\} .
\end{array}
\end{aligned}
$$

The following proposition is a direct result from Theorems $5-7$.

Proposition 9. Assume that

(*) for each $\varepsilon \in P$, there exist $\omega=\omega(\varepsilon) \in R$ and $M=$ $M(\varepsilon) \geq 1$ such that $\lambda>\omega$ implies $\lambda \in \rho(A(\varepsilon))$ and

$$
\left\|(\lambda I-A(\varepsilon))^{-n}\right\| \leq \frac{M}{(\lambda-\omega)^{n}}, \quad \text { for } \lambda>\omega, n \in N .
$$

Then, for $\varepsilon \in P$

(a) the part $A_{0}(\varepsilon)$ of $A(\varepsilon)$ in $X_{0}(\varepsilon)=\overline{D(A(\varepsilon))}$ generates a $C_{0}$-semigroup $T(t, \varepsilon)$ on $X_{0}(\varepsilon)$ satisfying

$$
\|T(t, \varepsilon)\| \leq M e^{\omega t},
$$

(b) $A(\varepsilon)$ generates a nondegenerate integrated semigroup $S(t, \varepsilon)$ on $X$ satisfying

$$
\begin{gathered}
\|S(t+r, \varepsilon) x-S(t, \varepsilon) x\| \leq\left(M \int_{t}^{t+r} e^{\omega \tau} d \tau\right)\|x\|, \\
\text { for } t \geq 0, x \in X,
\end{gathered}
$$

(c) $X_{0}(\varepsilon)=C^{1}=\{x \in X \mid S(t, \varepsilon) x$ is a continuously differentiable $X$-value function for $t \geq 0\}$,

(d) $T(t, \varepsilon)=S^{\prime}(t, \varepsilon)$ on $X_{0}(\varepsilon)$.

The following theorem is about the continuity in parameters of $C_{0}$-semigroup that is obtained in [4]. We will use this theorem to prove a theorem about the Cauchy problem (17).

Theorem 10 (see [4]). Assume that

(1) for each $\varepsilon \in P, A(\varepsilon)$ is densely defined; that is $\overline{D(A(\varepsilon))}=X$,

(2) $D(A(\varepsilon))=D$, for all $\varepsilon \in P$,

(3) there are constants $M \geq 1$ and $\omega \in R$ such that

$$
\left\|(\lambda I-A(\varepsilon))^{-n}\right\| \leq \frac{M}{(\lambda-\omega)^{n}}
$$$$
\text { for } \lambda>\omega, n \in N \text {, and all } \varepsilon \in P \text {, }
$$

(4) for each $x \in D, A(\varepsilon) x$ is continuous in $\varepsilon \in P$.

Then $(\lambda I-A(\varepsilon))^{-1} x$ is continuous in $\varepsilon$ for each $x \in X$. Thus, the $C_{0}$-semigroup $T(t, \varepsilon)$ generated by $A(\varepsilon)$ is strongly continuous in $\varepsilon \in P$, and the continuity is uniform on bounded $t$-intervals. In particular, for any $\varepsilon \in P, h \in \mathscr{P}$ with $\varepsilon+h \in P$, and for any $t_{0} \in[0, \infty)$,

$$
\begin{array}{r}
\sup _{0 \leq t \leq t_{0}}\|T(t, \varepsilon+h) x-T(t, \varepsilon) x\|=\circ(1) \\
\text { as }|h| \longrightarrow 0, \quad \text { for each } x \in X .
\end{array}
$$

Now we present the theorem about (17).

Theorem 11. Assume that (2)-(4) of Theorem 10 hold.

Then $u(t, \varepsilon)=S^{\prime}(t, \varepsilon) u_{0}$ and $t \geq 0$ is the unique classical solution of (17). Further, $u(t, \varepsilon)$ is continuous with respect to $\varepsilon$. In particular, for any $\varepsilon \in P, h \in \mathscr{P}$ with $\varepsilon+h \in P$, and for any $t_{0} \in[0, \infty)$,

$$
\sup _{0 \leq t \leq t_{0}}\|u(t, \varepsilon+h)-u(t, \varepsilon)\|=\circ(1) \quad \text { as }|h| \longrightarrow 0 .
$$

Proof. Because (3) of Theorem 10 holds, Proposition 9(b) indicates that $A(\varepsilon)$ generates a nondegenerate integrated semigroup $S(t, \varepsilon)$ on $X$. Thus, it follows from Theorem 8 that

$$
u(t, \varepsilon)=S^{\prime}(t, \varepsilon) u_{0}, \quad \text { for } u_{0} \in D
$$

is the unique classical solution of (17). 
From Proposition 9(d), we have $S^{\prime}(t, \varepsilon)=T(t, \varepsilon)$ on $X_{0}$, where $T(t, \varepsilon)$ is the $C_{0}$-semigroup that is generated by the part $A_{0}(\varepsilon)$ of $A(\varepsilon)$. Hence, we have

$$
u(t, \varepsilon)=S^{\prime}(t, \varepsilon) u_{0}=T(t, \varepsilon) u_{0}, \quad \text { for } u_{0} \in D .
$$

Because, for each $\varepsilon \in P, A_{0}(\varepsilon)$ is densely defined, that is $\overline{D\left(A_{0}(\varepsilon)\right)}=X_{0}(\varepsilon)$, Condition (1) of Theorem 10 is satisfied. Also (2)-(4) indicate that all other conditions of Theorem 10 are satisfied. Now, by applying Theorem 10, we have that the classical solution $u(t, \varepsilon)=T(t, \varepsilon) u_{0}$ is continuous with respect to parameter $\varepsilon$.

Next, we will discuss the nonhomogeneous abstract Cauchy problem

$$
\begin{gathered}
u^{\prime}(t)=A(\varepsilon) u(t)+f(t), \quad \text { for } t \geq 0, \\
u(0)=u_{0}
\end{gathered}
$$

where $\varepsilon$ is the parameter in $P$. For each $\varepsilon \in P$, the operator $A(\varepsilon)$ is a closed, linear, and nondensely defined operator on the Banach space $X . f:[0, \infty) \rightarrow X$ is continuously differentiable, and $f(0) \in X_{0}(\varepsilon)=\overline{D(A(\varepsilon))}$, for $\varepsilon \in P$.

In order to prove our main theorem for (27), we first state a result obtained in [4].

Theorem 12 (see [4]). Assuming that (2)-(4) of Theorem 10 are satisfied, then the integrated semigroup $S(t, \varepsilon)$ generated by $A(\varepsilon)$ is strongly continuous in $\varepsilon \in P$, and the continuity is uniform on bounded $t$-intervals. In particular, for any $\varepsilon \in P$, $h \in \mathscr{P}$ with $\varepsilon+h \in P$, and for any $t_{0} \in[0, \infty)$,

$$
\begin{array}{r}
\sup _{0 \leq t \leq t_{0}}\|S(t, \varepsilon+h) x-S(t, \varepsilon) x\|=\circ(1) \\
\text { as }|h| \longrightarrow 0, \quad \text { for each } x \in X .
\end{array}
$$

Now we present the main theorem about (27).

Theorem 13. Assume that (2)-(4) of Theorem 10 hold.

Then the unique classical solution $u(t, \varepsilon)$ of $(27)$ is continuous with respect to $\varepsilon$. In particular, for any $\varepsilon \in P, h \in \mathscr{P}$ with $\varepsilon+h \in P$, and for any $t_{0} \in[0, \infty)$,

$$
\sup _{0 \leq t \leq t_{0}}\|u(t, \varepsilon+h)-u(t, \varepsilon)\|=\circ(1) \quad \text { as }|h| \longrightarrow 0 \text {. }
$$

Proof. From (2) of Theorem 10 and Proposition 9(b), we have that $A(\varepsilon)$ generates a nondegenerate integrated semigroup $S(t, \varepsilon)$ on $X$. It follows from Theorem 8 that

$$
u(t, \varepsilon)=S^{\prime}(t, \varepsilon) u_{0}+\frac{d}{d t} \int_{0}^{t} S(t-\tau, \varepsilon) f(\tau) d \tau, \quad u_{0} \in D
$$

is the unique classical solution to (27).

Note that, from Proposition 9(d), we have $S^{\prime}(t, \varepsilon)=$ $T(t, \varepsilon)$ on $X_{0}$, where $T(t, \varepsilon)$ is the $C_{0}$-semigroup that is generated by the part $A_{0}(\varepsilon)$ of $A(\varepsilon)$. Since $u_{0} \in D \subset X_{0}$, $S^{\prime}(t, \varepsilon) u_{0}=T(t, \varepsilon) u_{0}$. Using the similar argument as that in the proof of Theorem 11, we see that $T(t, \varepsilon) u_{0}$ is continuous in $\varepsilon$. Thus, $S^{\prime}(t, \varepsilon) u_{0}\left(=T(t, \varepsilon) u_{0}\right)$ is continuous in $\varepsilon$.

Now we just need to show that $(d / d t) \int_{0}^{t} S(t-\tau, \varepsilon) f(\tau) d \tau$ is continuous in $\varepsilon$.

It is obvious that $S(t, \varepsilon) f(t)$ is continuous in $t$. Thus, we have

$$
\begin{aligned}
& \frac{d}{d t} \int_{0}^{t} S(t-\tau, \varepsilon) f(\tau) d \tau \\
& \quad=\frac{d}{d t} \int_{0}^{t} S(s, \varepsilon) f(t-s) d s \\
& \quad=S(t, \varepsilon) f(0)-S(0) f(-s)=S(t, \varepsilon) f(0)
\end{aligned}
$$

( since $S(0)=0)$.

It is sufficient to show that $S(t, \varepsilon) f(0)$ is continuous in $\varepsilon$. Note that Conditions (2)-(4) of Theorem 10 indicate that all assumptions of Theorem 12 are satisfied. Since $f(0) \in X$, it follows from Theorem 12 that $S(t, \varepsilon) f(0)$ is continuous in $\varepsilon$.

In summary, we have $u(t, \varepsilon)=S^{\prime}(t, \varepsilon) u_{0}+(d / d t) \int_{0}^{t} S(t-$ $\tau, \varepsilon) f(\tau) d \tau$ is continuous with respect to parameter $\varepsilon$.

\section{Application to a Parabolic Partial Differential Equation}

Consider the parabolic partial differential equation with boundary conditions

$$
\begin{gathered}
u_{t}=u_{x x}+k_{1} u_{x}+k_{2} u, \quad \text { for } t \geq 0, k_{1}, k_{2} \in R, \\
u(x, 0)=u_{0}(x), \quad \text { for } x \in[0,1], \\
u(0, t)=u(1, t)=0 .
\end{gathered}
$$

In this section, we will apply the abstract results obtained in Section 3 to show that the classical solution of (32) is continuous with respect to parameters $k_{1}$ and $k_{2}$.

We start with making the change in variable $v=e^{\left(k_{1} / 2\right) x} u$. Then $v$ satisfies the equation

$$
\begin{gathered}
v_{t}=v_{x x}+\left(k_{2}-\frac{k_{1}^{2}}{4}\right) v, \quad \text { for } t \geq 0, k_{1}, k_{2} \in R \\
v(x, 0)=v_{0}(x), \quad \text { for } x \in[0,1] \\
v(0, t)=v(1, t)=0
\end{gathered}
$$

where $v_{0}(x)=e^{\left(k_{1} / 2\right) x} u_{0}(x)$.

The abstract Cauchy problem formulated from (33) is

$$
\frac{d v(t)}{d t}=A(\varepsilon) v(t), \quad v(0)=v_{0}
$$

on $X=\left(C[0,1],\|\cdot\|_{\infty}\right), t \geq 0$, where

$$
\begin{gathered}
A(\varepsilon)=\frac{d^{2}}{d x^{2}}+B(\varepsilon), \quad \text { where } B(\varepsilon)=\left(k_{2}-\frac{k_{1}^{2}}{4}\right), \\
\varepsilon=\left(k_{1}, k_{2}\right) \in R^{2}, \\
D(A(\varepsilon))=\left\{v \in C^{2}[0,1] \mid v(0)=v(1)=0\right\} .
\end{gathered}
$$


Firstly, for each $\varepsilon$, the operator $A(\varepsilon)$ is not densely defined. Consider

$$
\overline{D(A(\varepsilon))}=X_{0}(\varepsilon)=\{v \in C[0,1] \mid v(0)=v(1)=0\} \subset X .
$$

However, the domains of $A(\varepsilon)$ are the same for all $\varepsilon$; that is $D(A(\varepsilon))=D$.

Secondly, it is easy to see that $A=d^{2} / d x^{2}$ with domain $D(A(\varepsilon))$ is a Hille-Yosida operator. In particular, for $\lambda>0$, consider the equation

$$
\lambda u-A u=v
$$

Then $\|u\|_{\infty}=\left|u\left(x_{0}\right)\right|$, where $0<x_{0}<1$. We may assume that $u\left(x_{0}\right)>0$. Then $u^{\prime \prime}\left(x_{0}\right) \leq 0$ and

$$
\lambda u\left(x_{0}\right) \leq \lambda u\left(x_{0}\right)-u^{\prime \prime}\left(x_{0}\right)=v\left(x_{0}\right) \leq\|v\|_{\infty} .
$$

Thus,

$$
\left\|(\lambda I-A)^{-1} v\right\| \leq \frac{1}{\lambda}\|v\|_{\infty} .
$$

Furthermore, since $B(\varepsilon)$ is bounded, then by the "bounded perturbations theorem" (see [11, page 76]), we have that $A(\varepsilon)=A+B(\varepsilon)$ is a Hille-Yosida operator on $X$ for each $\varepsilon \in R^{2}$. Now taking $P=\left\{\left(k_{1}, k_{2}\right)|| k_{1}\left|\leq k_{1 o},\right| k_{2} \mid \leq k_{2 o}\right\}$ for some $k_{1 o}, k_{2 o}>0$, we have that $A(\varepsilon)$ satisfies the uniform Hille-Yosida condition (*) of Proposition 9.

Thirdly, it is obvious that $A(\varepsilon) u=\left(d^{2} / d x^{2}\right) u+\left(k_{2}-k_{1}^{2} / 4\right) u$ is continuous with respect to $\varepsilon$ for each $u \in D$.

Therefore, from Theorem 11, it follows that the classical solution $v(x, t, \varepsilon)$ is continuous with respect to $\varepsilon$. Clearly, the classical solution $u(x, t, \varepsilon)=e^{-\left(k_{1} / 2\right) x} v(x, t, \varepsilon)$ of (32) is also continuous with respect to $\varepsilon$.

\section{Conflict of Interests}

The author declares that there is no conflict of interests regarding the publication of this paper.

\section{References}

[1] D. W. Brewer, "A parameter dependence problem in functionaldifferential equations," in Volterra and Functional-Differential Equations, vol. 81 of Lecture Notes in Pure and Applied Mathematics, pp. 187-195, Dekker, New York, NY, USA, 1982.

[2] R. Grimmer and M. He, "Fixed point theory and nonlinear periodic systems," The CUBO Mathematical Journal, vol. 11, no. 3, pp. 101-104, 2009.

[3] M. He, "A class of integrodifferential equations with memory," Semigroup Forum, vol. 73, no. 3, pp. 427-443, 2006.

[4] M. He, "On continuity in parameters of integrated semigroups," Discrete and Continuous Dynamical Systems A, pp. 403-412, 2003.

[5] C. Lizama, "On the convergence and approximation of integrated semigroups," Journal of Mathematical Analysis and Applications, vol. 181, no. 1, pp. 89-103, 1994.
[6] S. Nicaise, "The Hille-Yosida and Trotter-Kato theorems for integrated semigroups," Journal of Mathematical Analysis and Applications, vol. 180, no. 2, pp. 303-316, 1993.

[7] S. Busenberg and B. Wu, "Convergence theorems for integrated semigroups," Differential and Integral Equations, vol. 5, no. 3, pp. 509-520, 1992.

[8] H. R. Thieme, “'Integrated semigroups' and integrated solutions to abstract Cauchy problems," Journal of Mathematical Analysis and Applications, vol. 152, no. 2, pp. 416-447, 1990.

[9] W. Arendt, "Vector-valued Laplace transforms and Cauchy problems," Israel Journal of Mathematics, vol. 59, no. 3, pp. 327-352, 1987.

[10] G. Da Prato and E. Sinestrari, "Differential operators with nondense domain," Annali della Scuola Normale Superiore di Pisa, vol. 14, no. 2, pp. 285-344, 1987.

[11] A. Pazy, Semigroups of Linear Operators and Applications to Partial Differential Equations, vol. 44, Springer, New York, NY, USA, 1983. 


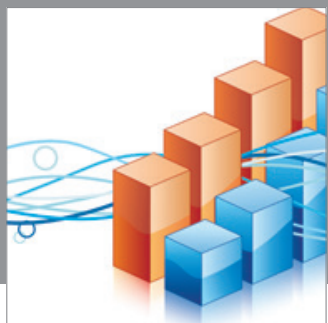

Advances in

Operations Research

mansans

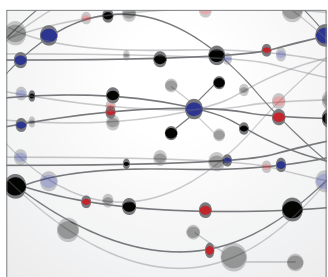

The Scientific World Journal
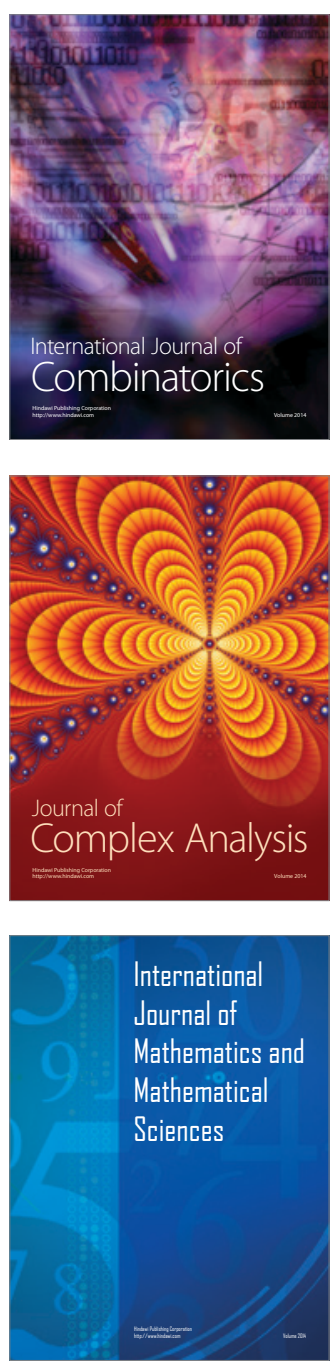
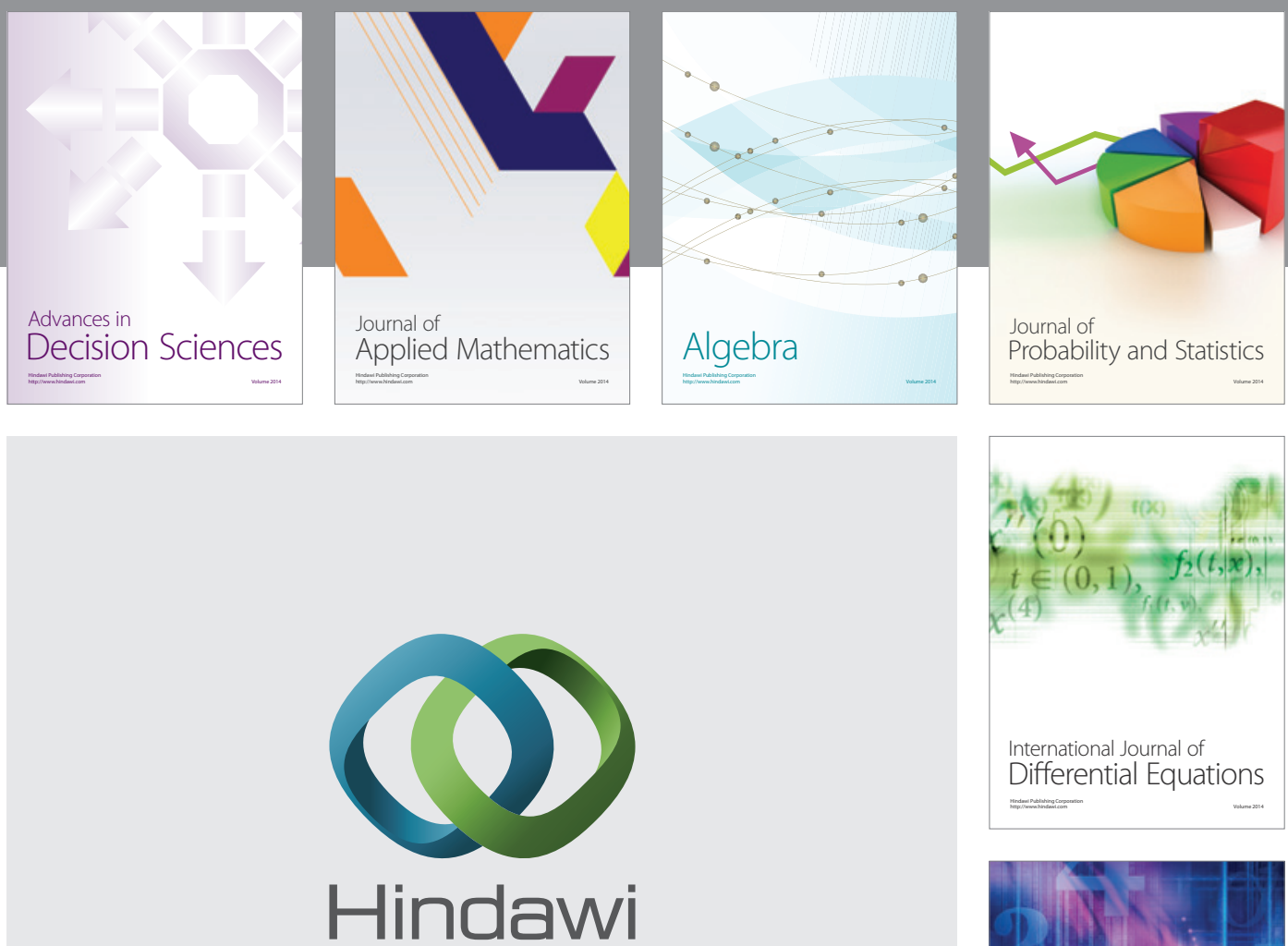

Submit your manuscripts at http://www.hindawi.com
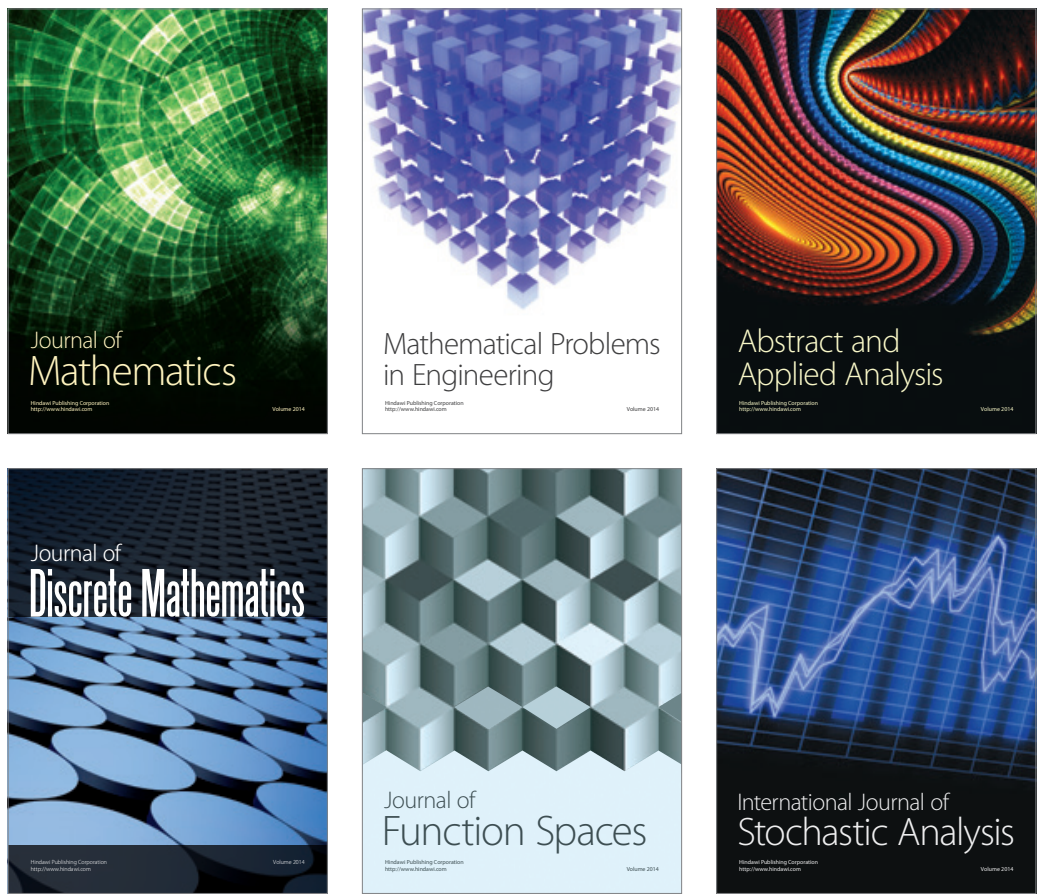

Journal of

Function Spaces

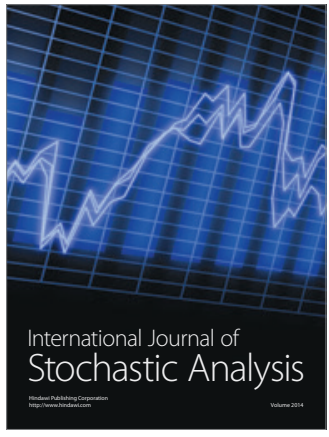

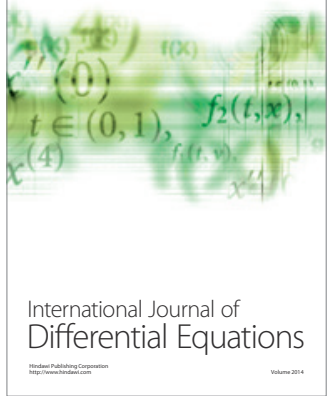
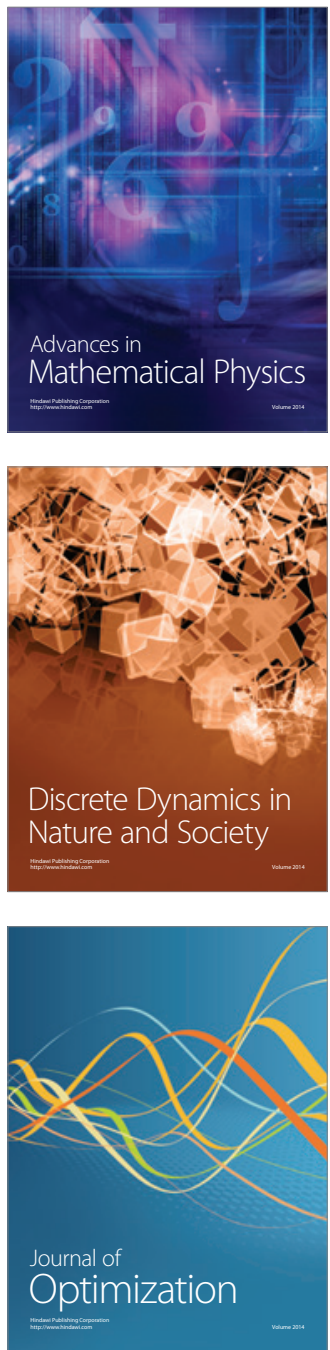